Document downloaded from:

http://hdl.handle.net/10251/37450

This paper must be cited as:

Sans Tresserras, JȦ.; Manjón Herrera, FJ.; Pereira, A.; Gómez-Tejedor, JA.; Monsoriu Soriano, JC. (2013). Oscillations studied with the smartphone ambient light sensor. European Journal of Physics. 34(6):1349-1354. doi:10.1088/0143-0807/34/6/1349.

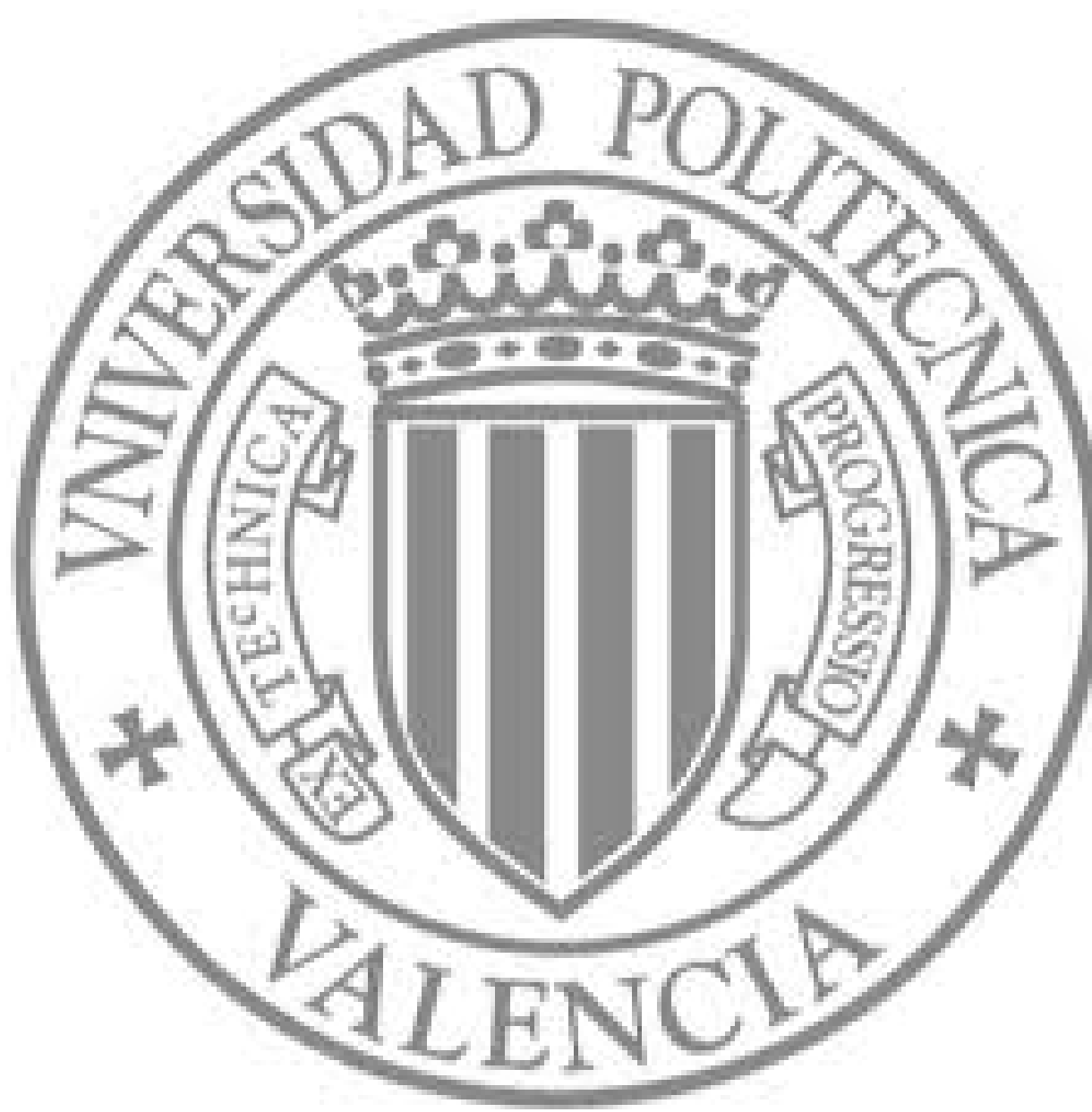

The final publication is available at

http://dx.doi.org/10.1088/0143-0807/34/6/1349

Copyright

IOP Publishing: Hybrid Open Access 


\title{
Oscillations studied with the smartphone ambient light sensor
}

\author{
Eur. J. Phys. 34 (2013) 1349-1354
}

doi:10.1088/0143-0807/34/6/1349

\author{
J. A. Sans ${ }^{1 *}$, F. J. Manjón' ${ }^{1}$ A. L. J. Pereira ${ }^{1}$, J. A. Gomez-Tejedor ${ }^{2}$, and \\ J. A. Monsoriu ${ }^{3}$ \\ 1 Instituto de Diseño para la Fabricación y Producción Automatizada, MALTA \\ Consolider Team, Universitat Politècnica de València, Camí de Vera s/n, 46022 València \\ (España) \\ ${ }^{2}$ Center for Biomaterials and Tissue Engineering, Universitat Politècnica de València, \\ Camí de Vera s/n, 46022 València (España) \\ ${ }^{3}$ Centro de Tecnologías Físicas, Universitat Politècnica de València, Camí de Vera s/n, \\ 46022 València (España) \\ *Email: juasant2@upv.es
}

\begin{abstract}
This paper makes use of a smartphone's ambient light sensor to analyze a system of two coupled springs undergoing either simple or damped oscillatory motion. Period, frequency, stiffness of the spring, damping constant, and extinction time are extracted from light intensity curves obtained using a free Android application. The results demonstrate the instructional value of mobile phone sensors as a tool in the physics laboratory.
\end{abstract}

Keywords: harmonic and damped oscillations, Android, smartphone light sensor, springs.

\section{Introduction}

Sensors found in common electronic devices can be used as a useful tool to bring students closer to measuring techniques in the physics laboratory. Digital cameras ${ }^{1}$, webcams ${ }^{2}$, optical computer mice ${ }^{3,4}$, Wii classic controller ${ }^{5}$, Xbox Kinect sensor ${ }^{6}$ and other video game consoles controllers ${ }^{7}$ have been used to carry out new laboratory experiments that allow visualizing basic concepts in Physics. Among those tools, the use of the Wiimote allows tracking several objects simultaneously by means of a Bluetooth connection and exploits the three accelerometers to follow them in 3D, those properties can be applied to a large amount of Physics experiments ${ }^{8}$. The use of the Xbox Kinect sensor allows measuring 3D positional data with a time base. However, these devices require specific software and are not widely available in the physics laboratory. On the other hand, the widespread use of mobile phones among young people and the constant evolution of the technology applied on these devices make them an attractive tool for possible application in scientific demonstrations and experimental measuremnets. 
Recently, Castro-Palacio et al. ${ }^{9,10}$ exploited the use of the Smartphone digital gyroscope to study a system of coupled oscillators. In this work we present a similar study but using the ambient light sensor of the smartphone. Light sensors are common devices on optical teaching Physics labs ${ }^{11}$, but use of their own phones to carry out the measurements increases the interest and motivation of the students performing the experiment. Here, we detail the experimental setup and the mathematical model used to analyze the variation of the recorded light intensity. The data acquired is used to determine important parameters describing the simple harmonic and damped oscillatory motion. The study of harmonic and damped oscillations is included in most syllabi of introductory physics courses, which are generally covered in the first courses of university degrees ${ }^{12}$.

\section{Basic theory}

The oscillatory movement along the $x$ axis of an object with mass $m$ coupled to two springs with stiffness $k$ and shifted with respect to the equilibrium position is described by the simple harmonic movement equations:

$$
\begin{gathered}
x(t)=A \sin \left(\omega_{0} t+\varphi\right) \\
T=\frac{2 \pi}{\omega_{0}}
\end{gathered}
$$

where $A$ is the shift amplitude, $\varphi$ is the initial phase and $\omega_{0}$ is the angular frequency, which is related to the oscillation period $T$ by equation 2 . Once the frequency of the simple harmonic movement is known, the spring constant $k$ can be calculated using the following expression:

$$
k=\frac{m \omega_{0}^{2}}{2}
$$

It is also well known that the light intensity is inversely proportional to the square of the distance between the detector and the light source assuming that one has a point light source whose power is spread in a spherical wave front. Therefore, if one places a light source in the same axis of the linear movement of the oscillatory system the intensity recorded by the light sensor is described by the equation:

$$
I(t)=I_{0} \frac{d_{0}^{2}}{\left(d_{0}+A \sin \left(\omega_{0} t+\varphi\right)\right)^{2}}
$$


where $I_{0}$ is the light intensity in the equilibrium position and $d_{0}$ is the distance between detector and the light sensor when the system is at the equilibrium position.

When the object of mass $m$ coupled to the springs is subjected to some kind of dissipative force, the oscillatory movement of the system is a damped oscillation described by the following equations:

$$
\begin{array}{r}
x(t)=B e^{-\gamma t} \sin (\omega t+\varphi) \\
\omega=\sqrt{\omega_{0}^{2}-\gamma^{2}}
\end{array}
$$

where $B$ is the oscillation amplitude, $\gamma$ is the damping constant, $\omega_{0}$ is the simple harmonic frequency, and $\omega$ is the damped oscillation frequency. In this case the light intensity measured by the light sensor in the same configuration as in the previous section, will be given by:

$$
I(t)=I_{0} \frac{d_{0}^{2}}{\left(d_{0}+B e^{-\gamma t} \sin (\omega t+\varphi)\right)^{2}}
$$

Therefore, similarly to the case of the previous subsection, we can obtain information about the oscillatory movement from the variation of the light intensity with the detector-source distance according to the equation 7.

\section{Experimental Set-Up}

The experimental set-up is shown in Figure 1. A glider is placed on an air track and connected to fixed ends of the track using two springs with the same stiffness. A smartphone is attached to the glider with the ambient light sensor facing the light source along the direction of the glider's motion. The smartphone used to carry out the experiment is a Samsung Galaxy SIII running version 4.0.4 of the Android operative system.

The variation of the light intensity measured by the smartphone as a function of time was recorded using the freely-available "Physics Toolbox Light Sensor" app ${ }^{13}$ and the acquired data analyzed with statistics software. The smartphone sensor was calibrated with a photometer. Using this experimental set-up we carried out two experiments to study simple harmonic and damped oscillatory motion. Both types of motion can be studied just by changing the glider's mass (adding or removing weight) and/or the air flux of the kinematic air track ${ }^{14}$. In this way it is possible to find the optimal conditions to study both types of movements. 


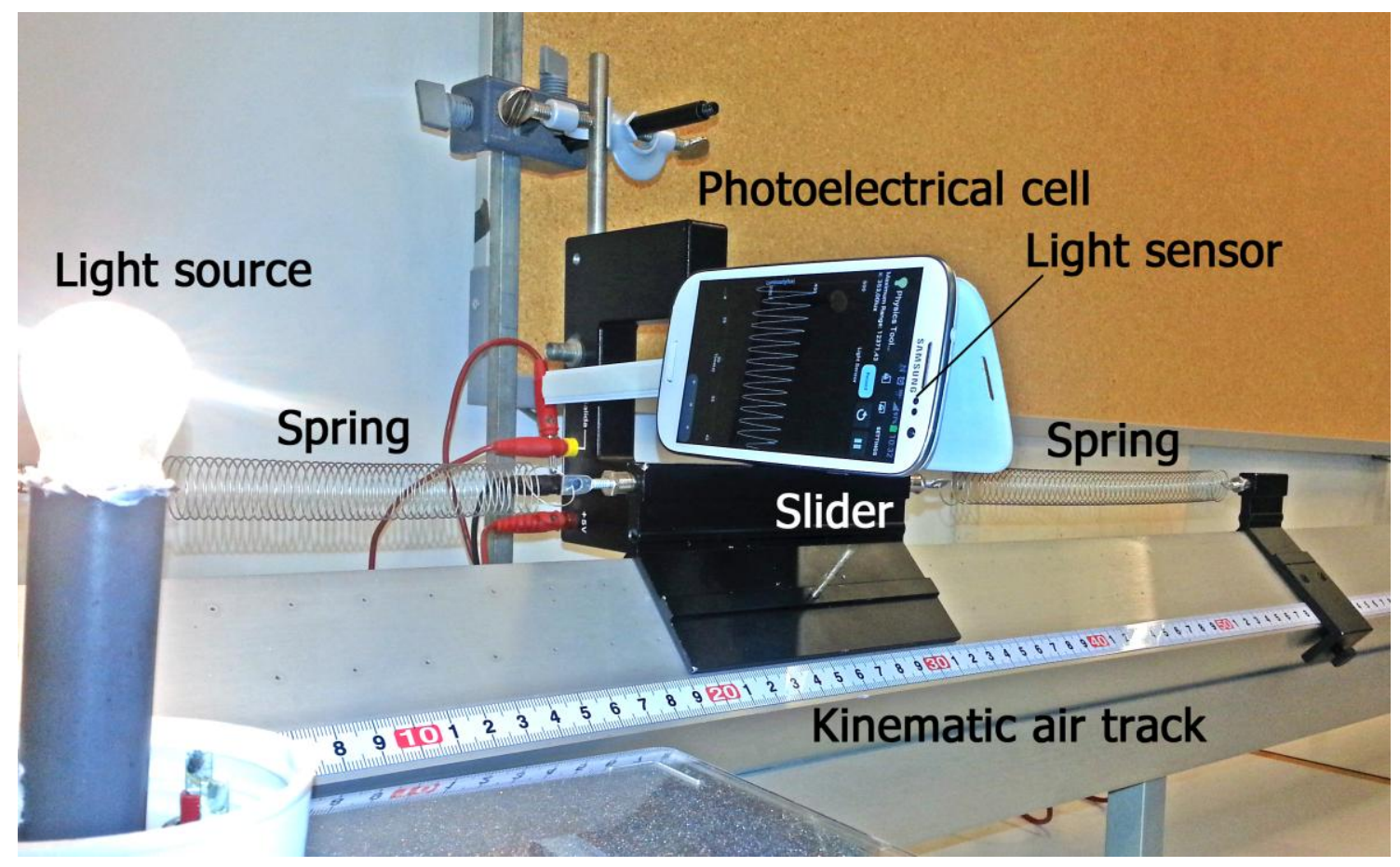

Figure 1. Picture of the coupled springs system set-up

\section{Results}

\subsection{Simple harmonic motion}

Using relatively low weights of the glider and/or relatively high air fluxes in the kinematic air track, we can obtain conditions closer to the friction-free movement so that the oscillating system describes a simple harmonic motion. Figure 2 shows the results of the recorded oscillations with the light sensor as a function of time for different system masses (glider with weights + smartphone).

Experimental data displayed in figure 2 show a weak damping that can be neglected as a first approximation. These data were fitted to equation 4 and a regression coefficient $R^{2}$ higher that 0.995 was obtained in all cases, thus indicating the good quality of the data. The oscillation frequencies (periods) obtained by these fits (Table I) decrease (increase) with the mass of the system. These period and frequency values are compared with those obtained from a photoelectric cell synchronized to a chronometer (Phywe). The good agreement between the values obtained by using both methods (in most cases below 1\%), provides confidence on the validity of the results obtained with the smartphone. 

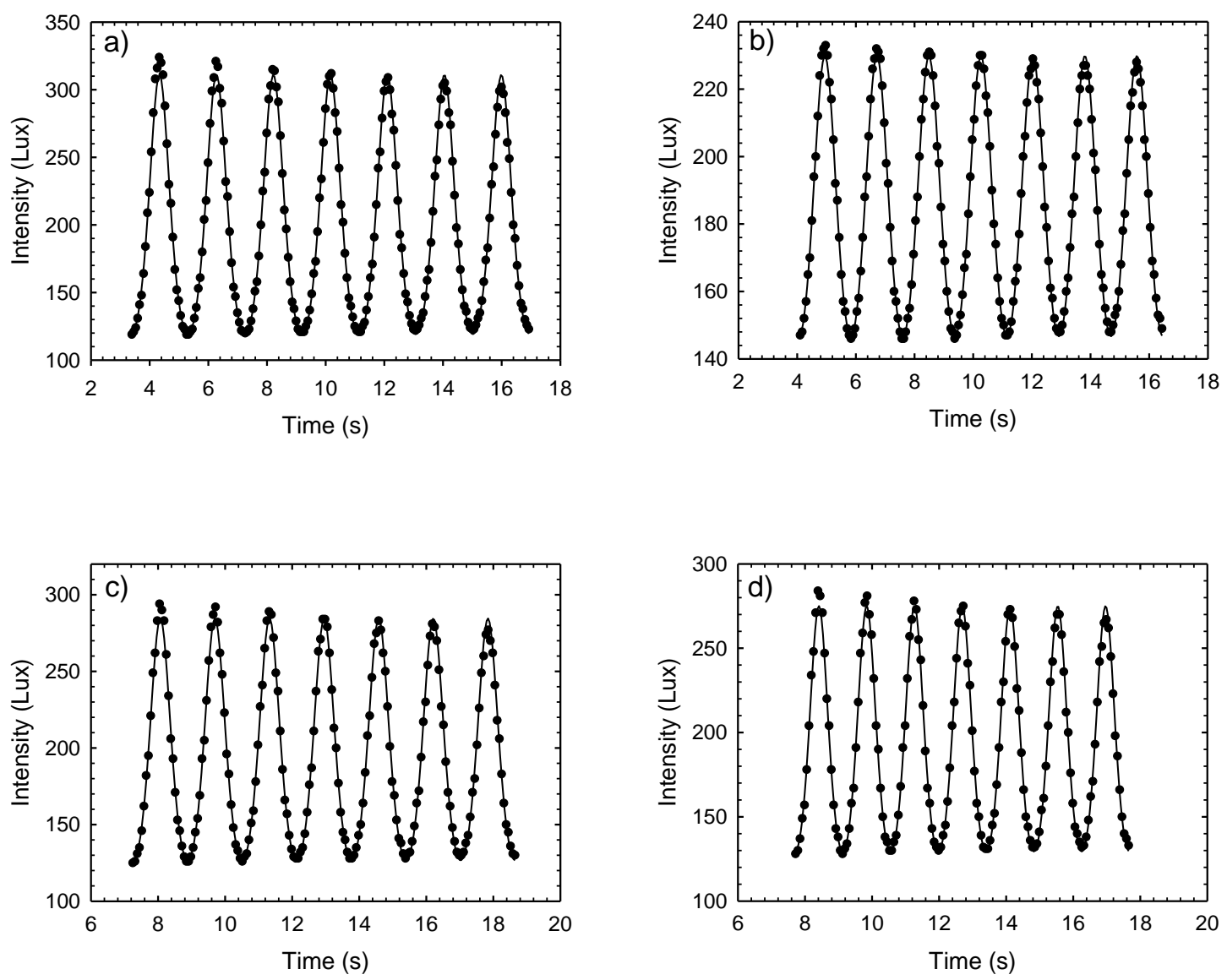

Figure 2. Light intensity versus time in a system with two coupled springs and with a mass of: a) $646 \mathrm{~g}$, b) $506 \mathrm{~g}$, c) $450 \mathrm{~g}$, and d) $310 \mathrm{~g}$.

The stiffness of the springs can be obtained by the so-called dynamical method which is based on the measurement of the time needed for the glider to carry out a determined number of oscillations. Equation 3 allows us to determine the stiffness of the springs, assuming that in our case both springs are identical. The values obtained for $k$ are compared (Table I) with the average value of the stiffness obtained by the static method in both springs separately. In the static method the spring is hold vertically and the elongation produced by different weights hanged on it is related to the stiffness by:

$$
L=\frac{g}{k} m+L_{0}
$$


Table 1. Frequencies, periods, and stiffnesses of the springs obtained with the smartphone (data fitted to equation 4) and those obtained with other methods (Phywe and static method)

\begin{tabular}{ccccccc}
\hline $\begin{array}{c}\text { Mass } \\
(\mathbf{k g})\end{array}$ & $\begin{array}{c}\boldsymbol{\omega}_{0} \\
\mathbf{p h o n e} \\
\left(\mathbf{s}^{-1}\right)\end{array}$ & $\begin{array}{c}\boldsymbol{T} \\
\text { phone } \\
(\mathbf{s})\end{array}$ & $\begin{array}{c}\boldsymbol{\omega}_{\boldsymbol{0}} \\
\text { Phywe } \\
\left(\mathbf{s}^{-1}\right)\end{array}$ & $\begin{array}{c}\boldsymbol{T} \\
\text { Phywe } \\
(\mathbf{s})\end{array}$ & $\begin{array}{c}\boldsymbol{k} \\
\text { phone } \\
(\mathbf{N} / \mathbf{m})\end{array}$ & $\begin{array}{c}\text { Static } \\
\text { method } \\
(\mathbf{N} / \mathbf{m})\end{array}$ \\
\hline & & & & & & \\
$0.646(1)$ & $3.2348(1)$ & $1.9424(1)$ & $3.242(1)$ & $1.938(2)$ & $3.380(7)$ & \\
$0.506(1)$ & $3.5442(1)$ & $1.7728(1)$ & $3.627(4)$ & $1.732(2)$ & $3.178(8)$ & $3.6(2)$ \\
$0.450(1)$ & $3.8584(1)$ & $1.6284(1)$ & $3.897(5)$ & $1.612(2)$ & $3.350(9)$ & \\
$0.310(1)$ & $4.4092(1)$ & $1.4250(1)$ & $4.39(3)$ & $1.431(2)$ & $3.013(10)$ & \\
\hline
\end{tabular}

\subsection{Damped oscillatory movement}

The increase of the mass of the slider and/or the reduction of the air flux in the kinematic track causes an increase of the action of the dissipative force between the slider and the track giving rise to a damped oscillatory movement. Figure 3 shows the results of the recorded oscillation as a function of time for different system masses using light sensor (glider with weights + smartphone).

The experimental data shown in figure 3 have been fitted using the equation 7 . The quality of the fit can be seen in the values of the regression coefficient $\mathrm{R}^{2}$, always higher than 0.98. Again, the high value of the $\mathrm{R}^{2}$ coefficient indicates the good quality of our experimental data. Table II shows the oscillation frequencies and the damping coefficients obtained by the fits for the different system masses with a constant air flux. It is noteworthy that the damping coefficient decreases with the increase of the system mass. Once the damping coefficient is known, it is possible to obtain the value of the oscillation extinction time using:

$$
\tau=\frac{1}{2 \gamma}
$$



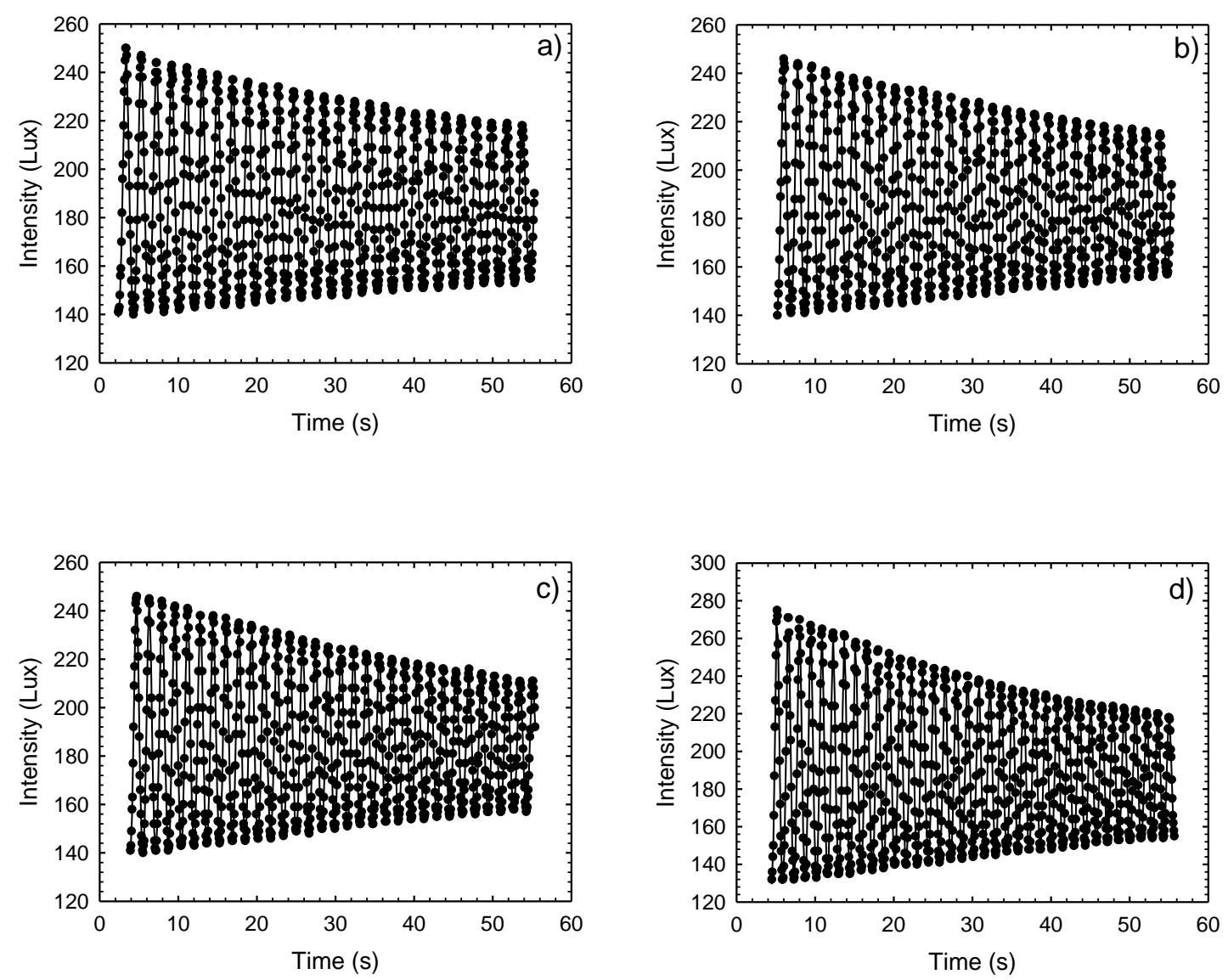

Figure 3. Light intensity versus time in a system with two coupled springs, with the action of the dissipative force, and with masses of: a) $646 \mathrm{~g}$, b) $506 \mathrm{~g}$, c) $450 \mathrm{~g}$, and d) $310 \mathrm{~g}$.

Table 2. Frequencies, damping time, stiffness of the springs, and extinction time obtained with the smartphone (data fitted to equation 7) and with other methods (static method)

\begin{tabular}{ccccccc}
\hline $\begin{array}{c}\text { Mass } \\
(\mathbf{k g})\end{array}$ & $\begin{array}{c}\boldsymbol{\rho} \\
\text { phone } \\
\left(\mathbf{s}^{-1}\right)\end{array}$ & $\begin{array}{c}\boldsymbol{p} \\
\text { phone } \\
\left(\mathbf{s}^{-1}\right)\end{array}$ & $\begin{array}{c}\omega_{0} \\
\text { phone } \\
\left(\mathbf{s}^{-1}\right)\end{array}$ & $\begin{array}{c}\tau \\
\text { phone } \\
(\mathbf{s})\end{array}$ & $\begin{array}{c}k \\
\text { phone } \\
(\mathbf{N} / \mathbf{m})\end{array}$ & $\begin{array}{c}\boldsymbol{k} \\
\text { method } \\
(\mathbf{N} / \mathbf{m})\end{array}$ \\
\hline $0.646(1)$ & $3.2433(1)$ & $0.010(2)$ & $3.2(6)$ & $50(10)$ & $3(1)$ & \\
$0.506(1)$ & $3.5485(1)$ & $0.012(2)$ & $3.5(6)$ & $41(7)$ & $3(1)$ & $3.6(2)$ \\
$0.450(1)$ & $3.8681(1)$ & $0.013(2)$ & $3.9(6)$ & $38(6)$ & $3(1)$ & \\
$0.310(1)$ & $4.4108(1)$ & $0.015(2)$ & $4.4(6)$ & $33(4)$ & $3(1)$ & \\
\hline
\end{tabular}


Finally, from equation 6 the value of the oscillation frequency corresponding to friction-free motion $\left(\omega_{0}\right)$ was obtained. This was, in turn, used to derive a value for the spring constant $k$, again assuming springs of identical stiffness (see Table II). It is clear from the data uncertainties in the determination of the spring constant from the damped oscillatory motion data are considerably larger than those associated with the analysis of simple harmonic motion.

\section{Conclusions}

We have successfully studied the harmonic and damped oscillations of a system with two coupled springs using the smartphone ambient light sensor and the Physics Toolbox Light Sensor Android app to record the light intensity as a function of time.

We have obtained the frequency, the period, the stiffness of the spring, the damping constant, and the extinction time of the simple harmonic and damped oscillatory movements. Results obtained for the frequency, the period, and the stiffness of the spring are in good agreement with those obtained using more traditional methods, and demonstrate the value of smartphone ambient light sensor as a tool in the undergraduate physics laboratory.

\section{Acknowledgements}

Authors would like to thank the Institute of Education Sciences, Universitat Politècnica de València (Spain), for the support of the Teaching Innovation Groups, e-MACAFI and MoMa.

\section{References}

1. J. A. Monsoriu, M. H. Giménez, J. Riera and A. Vidaurre, Eur. J. Phys. 26 (2005) 1149.

2. S. Shamim, W. Zia and M. S. Anwar, Am. J. Phys. 78 (2010) 433.

3. O. O. Romulo and K. N. Franklin, Am. J. Phys. 65 (1997) 1115.

4. T. W. Ng and K. T. Ang, Am. J. Phys. 73 (2005) 793.

5. S. L. Tomarken, D. R. Simons, R. W. Helms, W. E. Johns, K. E. Schriver et al., Am. J. Phys. 80 (2012) 351.

6. J. Ballester and Ch. Pheatt, Am. J. Phys. 81, 71 (2013).

7. M. Vannoni and S. Straulino, Eur. J. Phys. 28 (2007) 781.

8. A. Skeffington and K. Scully, Phys. Teach. 50 (2012) 482.

9. J. C. Castro-Palacio, L. Velazquez-Abad, F. Gimenez and J. A. Monsoriu, Eur. J. Phys. 34 (2013) 737. 
J. A. Sans et al., Eur. J. Phys. 34 (2013) 1349-1354

10. J. C. Castro-Palacio, L. Velazquez-Abad, M. H. Serrano and J. A. Monsoriu, Am. J. Phys. 81 (2013) 472.

11. P. J. Ouseph, K. Driver, and J. Conklin, Am. J. Phys. 69, 1166 (2001).

12. C. Kittel, W. D. Knight and M. A. Ruderman, Berkeley Physics Course, Vol. 1: Mechanics, McGraw-Hill, New York, (1973).

13. Vieyra software, https://play.google.com/store/apps

14. J. Berger, Eur. J. Phys. 9 (1988) 47. 